\title{
Numerical Analysis of Erosion Caused by Biomimetic Axial Fan Blade
}

\author{
Jun-Qiu Zhang, ${ }^{1}$ Zhi-Wu Han, ${ }^{1}$ Hui-Na Cao, ${ }^{1}$ Wei Yin, ${ }^{1}$ \\ Shi-Chao Niu, ${ }^{1}$ and Hui-Yuan Wang ${ }^{2}$ \\ ${ }^{1}$ Key Laboratory of Bionics Engineering of Ministry of Education, Jilin University, Changchun 130022, China \\ ${ }^{2}$ College of Materials Science and Engineering, Jilin University, Changchun 130022, China
}

Correspondence should be addressed to Zhi-Wu Han; zwhan@jlu.edu.cn

Received 29 September 2013; Accepted 27 November 2013

Academic Editor: S. Miyazaki

Copyright (C) 2013 Jun-Qiu Zhang et al. This is an open access article distributed under the Creative Commons Attribution License, which permits unrestricted use, distribution, and reproduction in any medium, provided the original work is properly cited.

\begin{abstract}
Damage caused by erosion has been reported in several industries for a wide range of situations. In the present work, a new method is presented to improve the erosion resistance of machine components by biomimetic method. A numerical investigation of solid particle erosion in the standard and biomimetic configuration blade of axial fan is presented. The analysis consists in the application of the discrete phase model, for modeling the solid particles flow, and the Eulerian conservation equations to the continuous phase. The numerical study employs computational fluid dynamics (CFD) software, based on a finite volume method. User-defined function was used to define wear equation. Gas/solid flow axial fan was simulated to calculate the erosion rate of the particles on the fan blades and comparatively analyzed the erosive wear of the smooth surface, the groove-shaped, and convex hull-shaped biomimetic surface axial flow fan blade. The results show that the groove-shaped biomimetic blade antierosion ability is better than that of the other two fan blades. Thoroughly analyze of antierosion mechanism of the biomimetic blade from many factors including the flow velocity contours and flow path lines, impact velocity, impact angle, particle trajectories, and the number of collisions.
\end{abstract}

\section{Introduction}

Wear is one of the main reasons for failures of mechanical parts. $[1,2]$ Erosion is an attractive branch in the domain of wear. Solid particle erosion is a dynamic process that occurs in different machine-parts due to the impingement of solid particles [3]. Centrifugal and axial fan working medium contains large amounts of solid particles, the particles impacting the surface of blade by very high speed and causing erosion of blade and housing; the erosion of blade is the most serious, often appear blade fracture, runaway and other major accidents. Hence, researches in material erosion including mechanism, factors of erosion, and the optimal selection of materials play a significant role in saving materials, reducing energy consumption, and improving economic efficiency. Inevitably, research on erosion is attracting more and more attention in the international academic circle [4-8]. It has been concluded by previous investigators that general methods used to reduce erosion are enhancing the wear resistance of material surface by means of wear resistant materials or coating material with better wear resistance [9-13].

Nature is a school for scientists and engineers; after billions of years of evolution, creatures in nature possess almost perfect structures and functions [14, 15]. In nature, some animals, for example, scorpion, are living in the sand and other gas/solid mixed-media environment, which exhibit excellent antierosion function under gas/solid mixed media environment [16]. Han et al. [17] showed that scorpions through the adaptation of the living environment and their own evolution, the formation of a special distribution of the convex and groove on the back, which can change the state of the surface boundary layer flow, and hence, reduce erosion of surface. Figure 1 illustrates the morphology of desert scorpion and biomimetic modeling.

Many of the factors which control the rate of erosion, such as particle velocity or particle mass flow rate, particle diameter, impact angle, and particle distribution can be studied at different flow conditions of the system. A lot of 


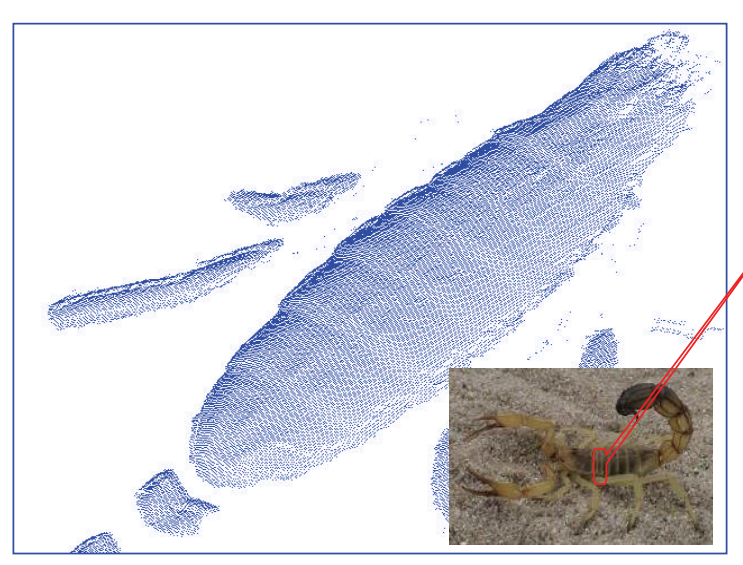

(a)

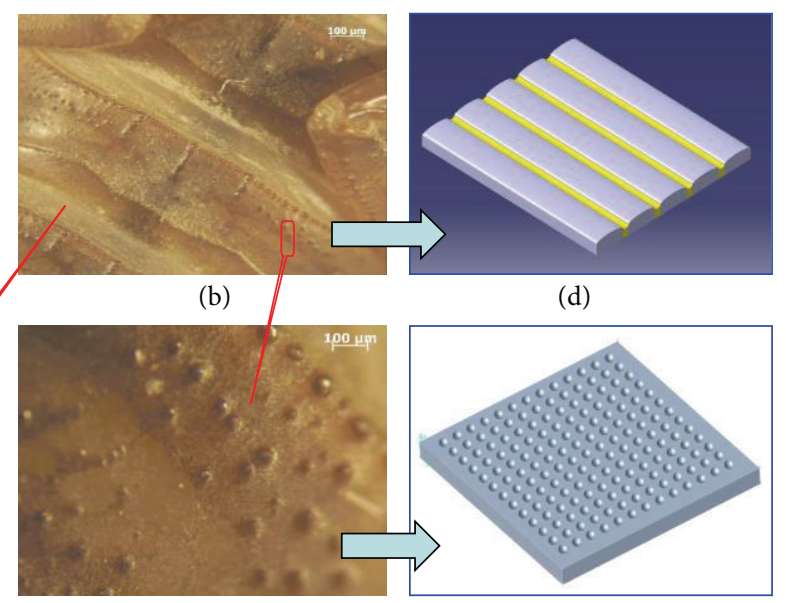

(c) (e)

FIGURE 1: The dorsal surface of the scorpion: (a) scanned data using a laser scanner; (b) the groove of scorpion back; (c) the convex hull of scorpion back; (d) bionic groove pattern; (e) bionic convex pattern.

practical examples may be found when a change in flow conditions has greatly increased or decreased erosion.

The experimental study on the dynamic behavior of solid particles requires special equipment and methodology to pursue this goal. Also, the erosion process is a complex problem to obtain a mathematical formula to account for some of the factors which control the rate of erosion of the blade. This paper presents a numerical study of the erosion process of biomimetic axial fan blade, applying computational fluid dynamics (CFD).

\section{Description of Analysis}

The numerical study of the erosion process applying CFD considers a mathematical model with Eulerian conservation equations in the continuous phase and a Lagrangian frame to simulate a discrete second phase. The dispersion of particles in the fluid phase can be predicted using a stochastic tracking model. This model includes the effect of instantaneous turbulent velocity fluctuations on the particle trajectories.

2.1. Governing Equations. The computational domain considers the mass conservation and momentum equations for incompressible flow in a $3 \mathrm{D}$ geometry in a steady state. The mass conservation is

$$
\nabla \cdot \rho v=S_{m}
$$

where $\nabla$ is the operator nabla, $\rho$ is the density $\left(\mathrm{kg} / \mathrm{m}^{3}\right), v$ is the velocity vector $(\mathrm{m} / \mathrm{s})$, and $S_{m}$ is the mass added to the continuous phase from the dispersed second phase. The momentum equation is

$$
\nabla \cdot(\rho v v)=-\nabla p+\nabla \cdot \tau+F
$$

where $p$ is the pressure on the fluid micro unit $(\mathrm{Pa}), \tau$ is the stress tensor, and $F$ are the forces that arise from interaction with the dispersed phase. For turbulence, the numerical study includes the standard $k-\varepsilon$ model [18], where the turbulent kinetic energy equation, $k$, is expressed by

$$
\frac{\partial\left(\rho \kappa u_{i}\right)}{\partial x_{i}}=\frac{\partial}{\partial x_{j}}\left[\left(\mu+\frac{\mu_{i}}{\sigma_{k}}\right) \frac{\partial k}{\partial x_{j}}\right]+G_{k}-\rho \varepsilon,
$$

and the dissipation rate equation, $\varepsilon$, is expressed by

$$
\frac{\partial\left(\rho \varepsilon u_{i}\right)}{\partial x_{i}}=\frac{\partial}{\partial x_{j}}\left[\left(\mu+\frac{\mu_{t}}{\sigma_{\varepsilon}}\right) \frac{\partial k}{\partial x_{j}}\right]+C_{1 \varepsilon} \frac{\varepsilon}{k} G_{K}-C_{2 \varepsilon} \rho \frac{\varepsilon^{2}}{k},
$$

where $u_{i}$ is the velocity on the direction $i(\mathrm{~m} / \mathrm{s}), x_{i}$ is distance coordinate on the direction $i(\mathrm{~m}), x_{j}$ is distance coordinate on the direction $j(\mathrm{~m}), \mu$ is molecular dynamic viscosity $(\mathrm{kg} / \mathrm{m}$ $\mathrm{s}), \mu_{i}$ is molecular dynamic viscosity on the direction $i(\mathrm{~kg} / \mathrm{m}$ s), $G_{k}$ represents the generation of turbulent kinetic energy due the mean velocity gradients, $C_{1 \varepsilon}$ and $C_{2 \varepsilon}$ are constants $\left(C_{1 \varepsilon}=1.44, C_{2 \varepsilon}=1.92\right)$, and $\sigma_{k}, \sigma_{\varepsilon}$ are the turbulent Prandtl numbers for $k$ and $\varepsilon\left(\sigma_{k}=1.0, \sigma_{\varepsilon}=1.3\right)$, respectively.

2.2. Discrete Phase Model (DPM). This model permits us to simulate a discrete second phase in a Lagrangian frame of reference, where the second phase consists of spherical particles dispersed in the continuous phase. The coupling between the phases and its impact on both the discrete phase trajectories and the continuous phase flow is included. The turbulent dispersion of particles is modeled using a stochastic discrete-particle approach. This approach predicts the turbulent dispersion by integrating the trajectory equations for individual particles, using the instantaneous fluid velocity. The prediction of particle dispersion makes use of the concept of the integral time scale, $T_{L}$, which describes the time spent in turbulent motion along the particle path. This time scale can be approximated in the standard $k-\varepsilon$ model as

$$
T_{L} \approx 0.15 \frac{k}{\varepsilon}
$$



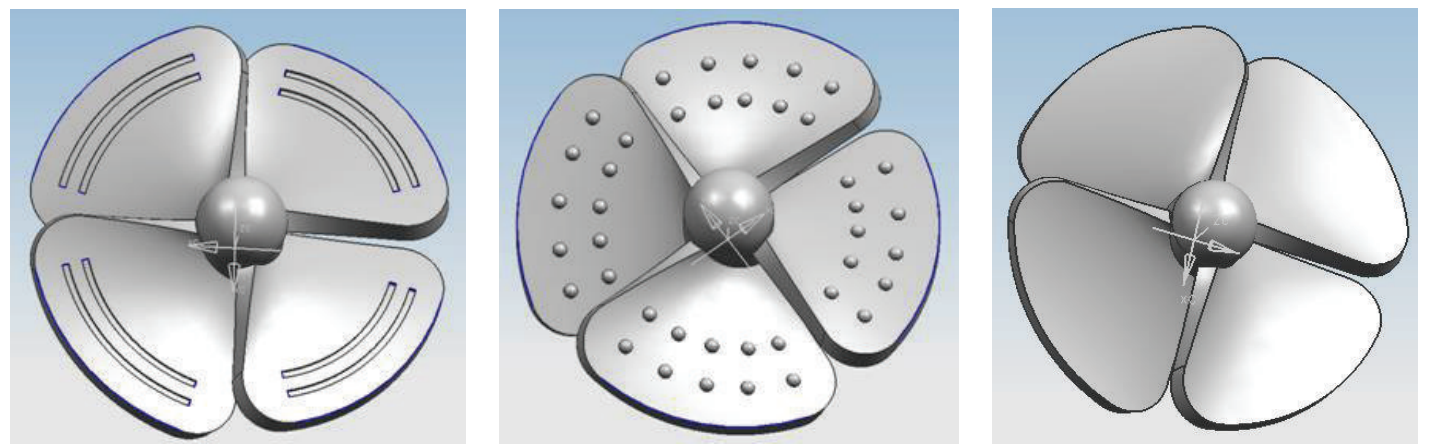

FIgURE 2: The geometrized structure graph of axial fan blades.

The trajectory of a discrete phase particle can be predicted by integrating the force balance on the particle, which is written in a Lagrangian reference frame. This force balance equates the particle inertia with the forces acting on the particle:

$$
\frac{d_{u_{p}}}{d t}=F_{D}\left(u-u_{p}\right)+F_{\text {other }},
$$

where $u_{p}$ is the particle velocity $(\mathrm{m} / \mathrm{s}), u$ is the velocity $(\mathrm{m} / \mathrm{s})$, $F_{D}$ is drag force $(\mathrm{N}), F_{D}\left(u-u_{p}\right)$ is the drag force per unit particle mass, $F_{\text {other }}$ is the additional forces $(\mathrm{N})$, and $F_{D}$ is defined as

$$
F_{D}=\frac{18 \mu}{\rho_{d} d_{p}^{2}} \frac{C_{D} R_{e}}{24},
$$

where $C_{D}$ is the drag coefficient is applied for smooth spherical particles, $R_{e}$ is the relative Reynolds number, and $d_{p}$ is the particle diameter $(\mathrm{m})$. The additional forces, $F_{\text {other }}$, in this case are compounded by the force required to accelerate the fluid surrounding the particle, defined as

$$
F_{\text {other }}=\frac{1}{2} \frac{\rho}{\rho_{p}} \frac{d}{d t}\left(u-u_{p}\right)
$$

where $\rho_{p}$ is the apparent density of the particles, which is important when $\rho>\rho_{p}$, and an additional force due to the pressure gradient in the fluid is

$$
F_{\text {other }}=\frac{\rho}{\rho_{p}} u_{p} \frac{\partial u}{\partial x} .
$$

The relative Reynolds number, $R_{e}$, is defined as

$$
R_{e}=\frac{\rho d_{p}\left(u_{p}-u\right)}{\mu} .
$$

To incorporate the effect of the discrete phase trajectories on the continuum, it is important to compute the interphase exchange of momentum from the particle to the continuous phase. This exchange is computed by examining the change in momentum of a particle as it passes through each control volume in the computational domain.
This momentum change is computed as

$$
F=\sum\left(\frac{18 \mu}{\rho_{d} d_{p}^{2}} \frac{C_{D} R_{e}}{24}\left(u_{p}-u\right)+F_{\text {other }}\right) \dot{m}_{p} \Delta t .
$$

Finally, to evaluate the erosion rate at the wall of the blade, applying the discrete phase model, it is important to define parameters such as the mass flow rate of the particle stream, $\dot{m}_{p}$, impact angle of the particle path with the wall face $\alpha$, function of the impact angle $f(\alpha)$, and the area of the wall face where the particle strikes the boundary $A_{\text {face }}$. The erosion rate is defined as

$$
R_{\text {erosion }}=\sum_{p=1}^{N_{\text {particle }}} \frac{\dot{m}_{p} C\left(d_{p}\right) f(\alpha) \nu^{b(v)}}{A_{\text {face }}},
$$

where $C\left(d_{p}\right)$ is a function of particle diameter, $\alpha$ is the impact angle of the particle path with the wall face, $f(\alpha)$ is a function of impact angle, $v$ is the relative particle velocity, $b(\nu)$ is a function of relative particle velocity, and $A_{\text {face }}$ is the area of the cell face at the wall. In (12) the impact angle function was defined by a piece-linear profile and the diameter function and velocity exponent function are $1.8 e-$ 09 and 2.6, respectively [18].

2.3. Modeling of Axial Fan. Geometric construction and meshing were performed with UG and GAMBIT. Bionic configuration created on the curved surface created by the projection along the direction of the normal surface, surface bias, and so forth. Figure 2 shows the geometrized structure graph of axial fan blades.

2.4. Mesh Generation. Different mesh type and size were used in each region, owing to the structure of each part of the axial fan, and the flow patterns are different. Longer segment structure of the inlet and outlet is simple, and the flow is relatively stable; hence, hexahedral grid was selected in the two regions. Strong rotation in the blade regional airflow, the flow is quite complex. Meanwhile, the structure of the leaves is more complex, especially the bionic blade. Therefore, selection of unstructured grid geometry structure strong adaptability, and leaves of the surface of the grid are encrypted. Table 1 is the results of mesh generation. 
TABLE 1: Each computational grid information of centrifugal fan.

\begin{tabular}{lccccc}
\hline Region of fan & $\begin{array}{c}\text { Lengthened segment } \\
\text { of air inlet }\end{array}$ & $\begin{array}{c}\text { Lengthened segment } \\
\text { of air outlet }\end{array}$ & Smooth surface & $\begin{array}{c}\text { Groove surface } \\
\text { morphology }\end{array}$ & $\begin{array}{c}\text { Convex surface } \\
\text { morphology }\end{array}$ \\
\hline Grid type & Hexahedral grid & Hexahedral grid & $\begin{array}{c}\text { Tetrahedron grid } \\
\text { refinement }\end{array}$ & $\begin{array}{c}\text { Tetrahedron grid } \\
\text { refinement }\end{array}$ & $\begin{array}{c}\text { Tetrahedron grid } \\
\text { refinement }\end{array}$ \\
\hline Cells & 223816 & 498200 & 2000671 & 2089127 & 2070634 \\
\hline
\end{tabular}

2.5. Calculation Model and Boundary Conditions. Pressureinlet boundary condition was used in the entrance of fan. Pressure outlet boundary condition was used in the exit of fan. The definition of turbulence parameters is based on turbulence intensity and hydraulic diameter.

Air flows in the tunnel with entrained solid particles at $11.6 \mathrm{~m} / \mathrm{s}$ velocity. The injection type was set to surface. Solid particles with $1500 \mathrm{~kg} / \mathrm{m}^{3}$ density were released from the inlet with an initial velocity of $11.6 \mathrm{~m} / \mathrm{s}$ assuming no slip between the particle and fluid. The particle diameters were $20 \mu \mathrm{m}, 50 \mu \mathrm{m}, 100 \mu \mathrm{m}, 150 \mu \mathrm{m}$, and $300 \mu \mathrm{m}$. And the mass flow rate was $2 \mathrm{~kg} / \mathrm{s}$. Internal flow field of fan was assumed to incompressible steady flow. RNG $k-\varepsilon$ model was used as turbulence model. Standard wall function method was used near the wall. Solid wall boundary was assumed no slip, and wall roughness is 0.5 . The interface boundary condition was applied for interface of fluid region. SIMPLEC algorithm was used for velocity-pressure coupling. The differential equations were discretized by a first-order upwind differencing scheme. A convergence criterion of $10^{-3}$ for each scaled residual component was specified for the relative error between two successive iterations.

Open the two-phase coupling calculation in the settings panel of discrete phase model. The boundary of reflect was applied for the wall and rebound model by using (13). Calculating model of erosion rate by using (14), the model for coal ash particles impacting steel was achieved through UDF:

$$
\begin{aligned}
& \frac{V_{n_{2}}}{V_{n_{1}}}=1.0-0.4159 \beta_{1}-0.4994 \beta_{1}^{2}+0.292 \beta_{1}^{3}, \\
& \frac{V_{t_{2}}}{V_{t_{1}}}=1.0-2.12 \beta_{1}+3.0775 \beta_{1}^{2}-1.1 \beta_{1}^{3},
\end{aligned}
$$

where $V_{n}$ and $V_{t}$ are the normal component and tangential component of particle collision surface speed, respectively, with the unit of $\mathrm{m} / \mathrm{s}$. The subscript 1,2 , respectively, denotes the amount before and after the collision. $\beta_{1}$ is the angle between the particles speed and the surface tangent before the collision, and $\beta_{2}$ is rebound angle of particles after the collision, and the unit is rad.

Experimental measurements reported by Hamed et al. $[19,20]$ indicated that erosion of a target material was found to be dependent upon the particle impact velocity and its impingement angle. Experimental measurements were obtained for coal ash particles impacting steel at different impacting velocities and impingement angles. The experimental data was used to establish the following empirical equation for the erosion mass parameter, $M$, which is defined as the ratio of the eroded mass of the target material to the mass of the impinging particles:

$$
\begin{gathered}
M=K_{1} R_{w}{ }^{2} V_{1}^{2} \cos ^{2} \beta_{1}\left(1-R_{T}^{2}\right)+K_{3}\left(V_{1} \sin \beta_{1}\right)^{4}, \\
R_{w}=1+C_{k}\left(K_{12} \sin \left(\frac{90}{\beta_{0}} \beta_{1}\right)\right), \\
R_{T}=1-0.0016 V_{1} \sin \beta_{1},
\end{gathered}
$$

where $V_{P}$ and $\beta_{P}$ are the impact velocity $(\mathrm{m} / \mathrm{s})$ and impingement angle $\left({ }^{\circ}\right)$, respectively. $\beta_{0}$ is maximum erosion angle $\left(^{\circ}\right)$, $R_{T}$ is tangential recovery ratio. The following values were used for the variables in (14) for $\beta_{0}=25^{\circ}$ (angle of maximum erosion):

$$
\begin{array}{ll}
C_{k}=1 & \beta_{1} \leq 2 \beta_{0} \\
C_{k}=0 & \beta_{1}>2 \beta_{0}
\end{array}
$$

with $K_{1}, K_{2}$, and $K_{3}$ material constants which are found to be $K_{1}=0.5225 \times 10^{-5}, K_{2}=0.266799, K_{3}=0.549 \times 10^{-12}$, and $\beta_{0}=25^{\circ}$.

\section{Results and Discussion}

3.1. Numerical Simulation Results. The simulation results of the erosion rate of the three kinds of blades are shown in the form of histogram in Figure 3. It was observed that the bionic blades exhibited better erosion resistance than the smooth surface blades. The groove surface blades showed the best erosion resistance compared to the other two kinds of blades. It is concluded that the best condition to improve the components erosion resistance is using the groove surface morphology.

3.2. Gas-Phase Flow Analysis. Figures 4, 5, and 6 show the flow velocity contours and flow path lines of the surface region ( $X=0$ cross-section) of the three types blades. As can be seen from the velocity contours, the flow velocities are higher around the smooth surface than those of the convex surface and the groove surface. Especially in the groove channel, the flow velocity was significantly lower than that of the smooth surface. Compared to the flow path lines, it is shown that the flow path lines on the smooth surface were smooth. On the convex surface were changed to a certain degree, it is indicated that the air flow was disturbed by the convex hull. But in the groove channel, the groove surface has a great influence on the airflow. The air was rotating in the groove channel, forming a stable low-speed reverse flow zone $[16,21]$. 


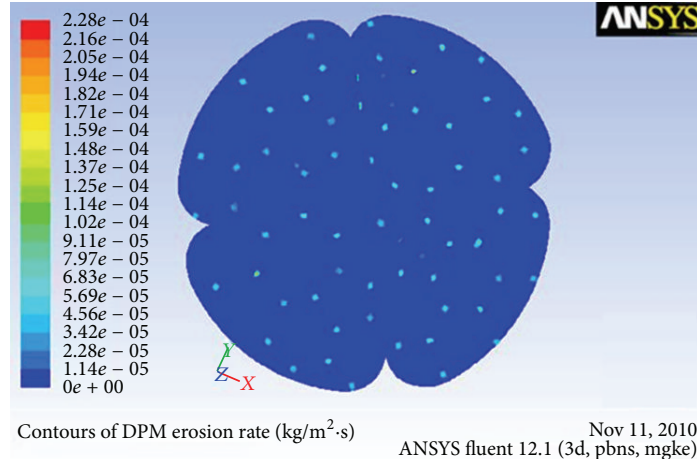

(a)

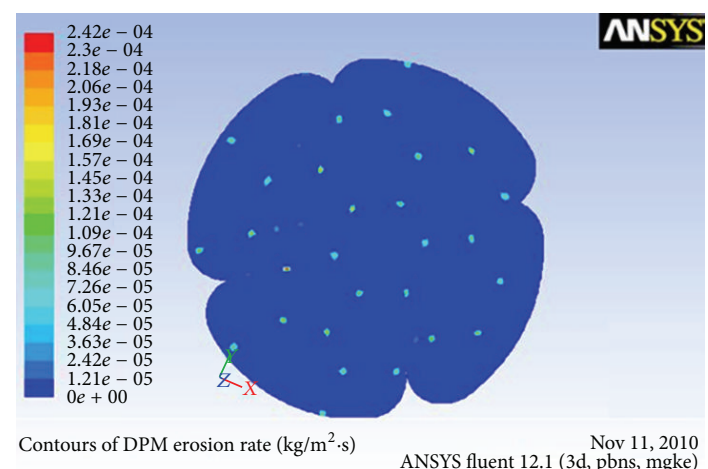

(b)

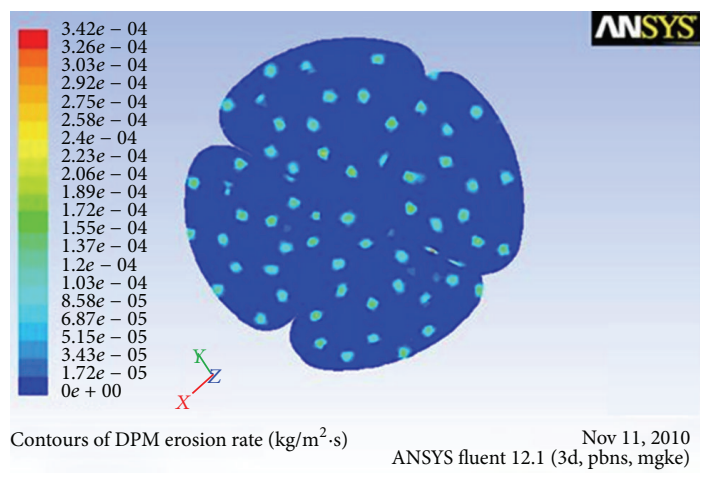

(c)

Figure 3: Numerical simulation results of the erosion rate of three kinds of blades: (a) groove surface morphology; (b) convex surface morphology; (c) smooth surface.
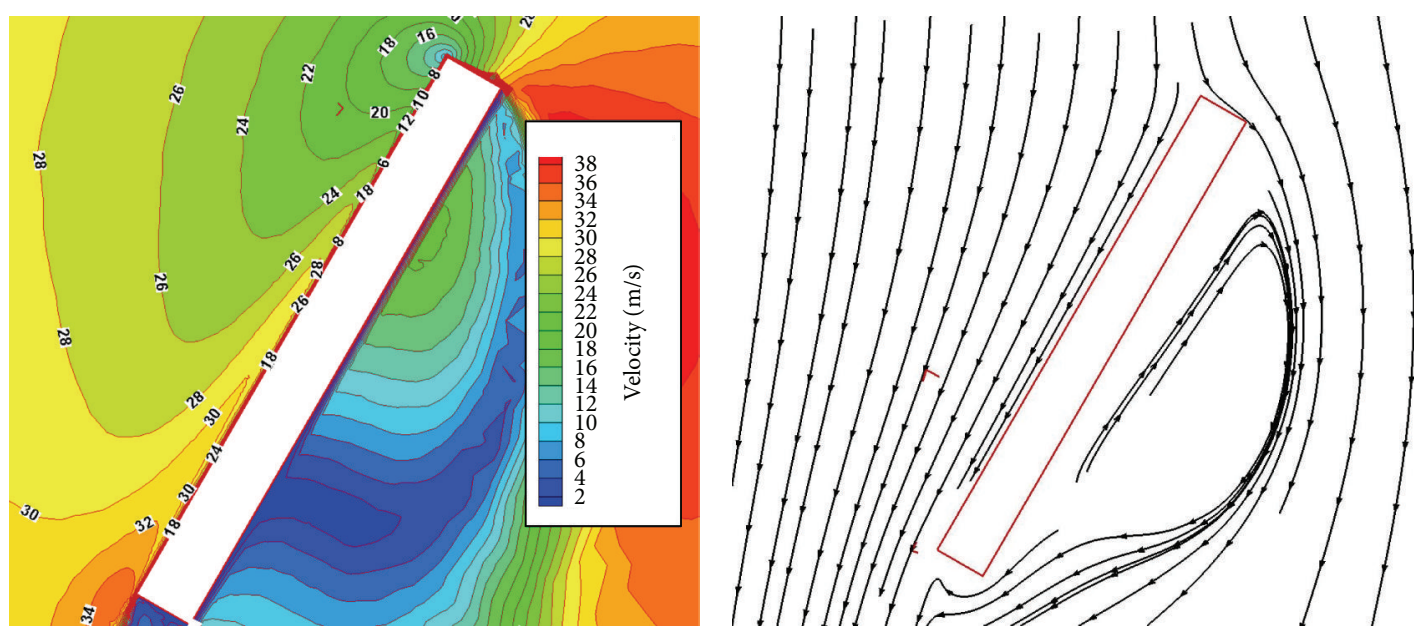

FIGURE 4: Flow velocity contours and path lines of the surface region of smooth surface.

The special flow pattern in the groove has significant influence on the erosion resistance of groove surface. The rotating flow in the groove plays an "air cushion" effect. On the one hand, the grooves can enhance fluid turbulence, which lead to the change of the flow field around the groove surface, and the particle motion pattern was changed subsequently. Some of the particles will leave the surface along with air flow without impact, and these particles would impact the surface if the surface was smooth. Therefore, the number of particles impacting the surface was decreased. On the other hand, as a result of the decrease the flow velocity and the velocities of the particles in the two-phase flow were decreased as well. The rotating flow in the groove can absorb particle energy which is used for impacting, and the energy 

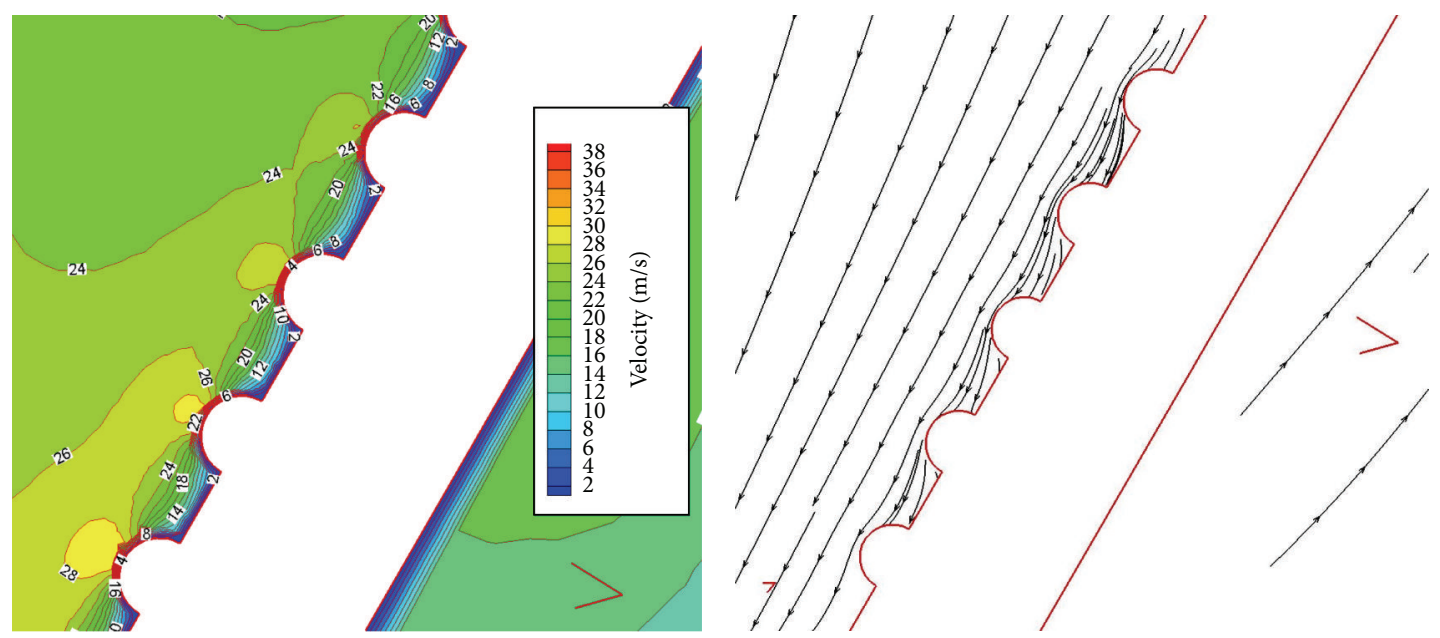

FIGURE 5: Flow velocity contours and path lines of the surface region of convex surface.
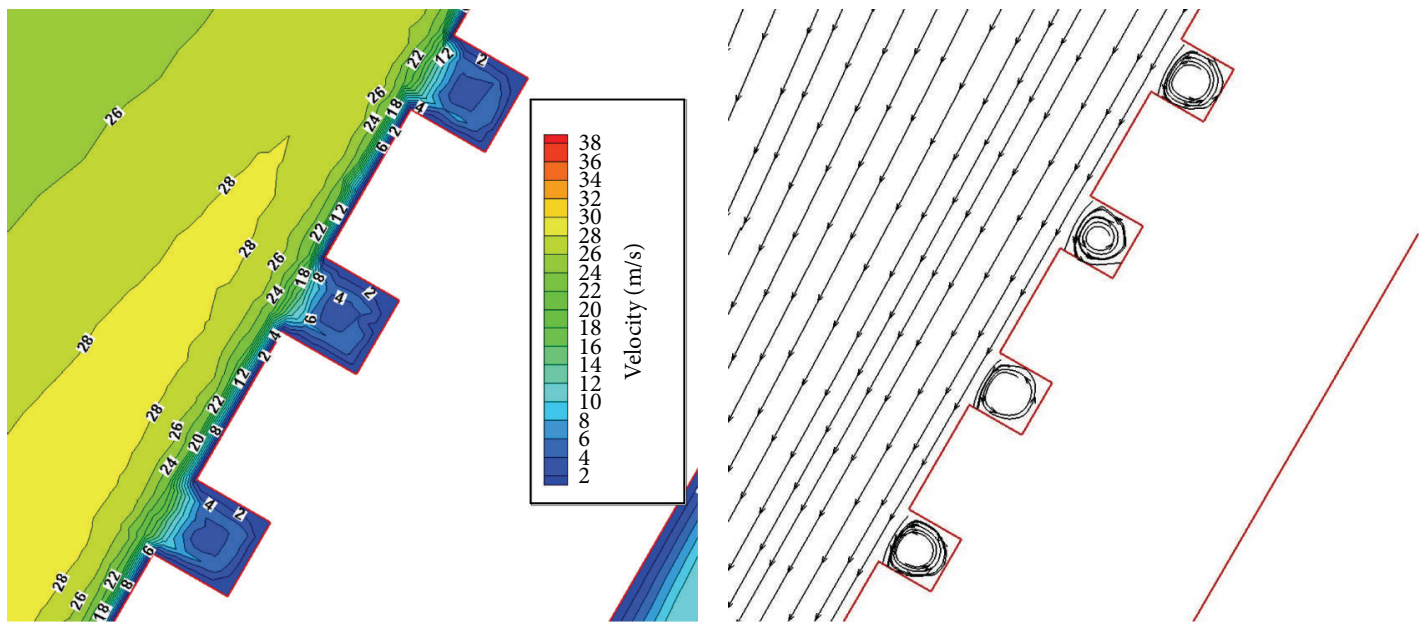

FIGURE 6: Flow velocity contours and path lines of the surface region of groove surface.

used in impact was correspondingly reduced. These features all help to reduce the particle impact damage on the blade surface and reduce erosion wear [21].

3.3. Particles Impact Velocity Analysis. The particle impact velocity is an important impingement variable which influences the erosion behavior of materials. The dependence of erosion rate $(E)$ on impact velocity $(v)$ is expressed by the following equation:

$$
E=k V^{n}
$$

where $n$ is velocity exponent and $k$ is a constant. The velocity exponent is usually in the range of 2-3 for ductile materials, while, for brittle materials, it can be much higher. Hence, it is clear that the erosion rate increases significantly with increase in the impact velocity.

In the present work, the particle impact velocity distributions on the surface of the three types of blades were analyzed. The particle impact velocities were obtained by the FLUENT postprocessing system. The impact velocities were recorded in the interval value of 2 units. Figure 7 shows the impact velocity distribution on the blade surface. For the smooth surface, the impact velocities are concentrated in $30 \mathrm{~m} / \mathrm{s}$. For the convex surface, the impact velocities are in the range of $5-35 \mathrm{~m} / \mathrm{s}$, and mainly distributed in the range of $25-30 \mathrm{~m} / \mathrm{s}$ with the probability of this velocity being greater than $80 \%$. But for the groove surface, the impact velocities are mostly concentrated in several fixed values, such as $10 \mathrm{~m} / \mathrm{s}, 16 \mathrm{~m} / \mathrm{s}$, and $30 \mathrm{~m} / \mathrm{s}$. There is a certain probability of impact velocities in $10 \mathrm{~m} / \mathrm{s}$ and $16 \mathrm{~m} / \mathrm{s}$. As a result, the particle impacts on the groove surface were occurred at the lower impact velocities compared with the smooth and convex surfaces, which lead to lower erosion than that of the other two types of surfaces [16].

3.4. Particles Impact Angle Analysis. Particle impact angle has an important effect on the erosion rate. The maximum erosion of ductile material occurs at angles between $20-30^{\circ}$ [17]. The probability of particle impact angle distribution on the blade surface is shown in Figure 8. On the smooth 


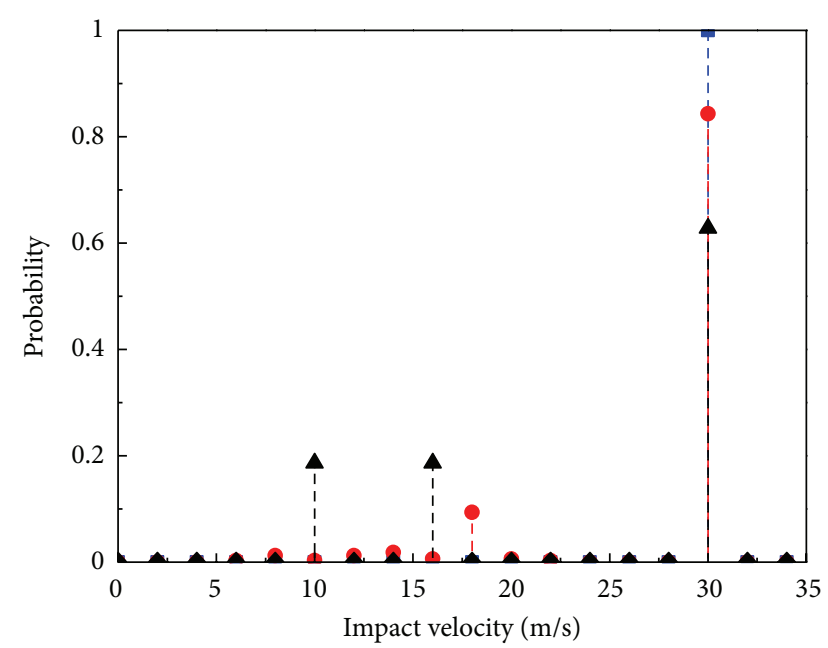

- Smooth blade

- Convex blade

$\Delta$ Groove blade

FIGURE 7: Particle-surface impact velocity distribution.

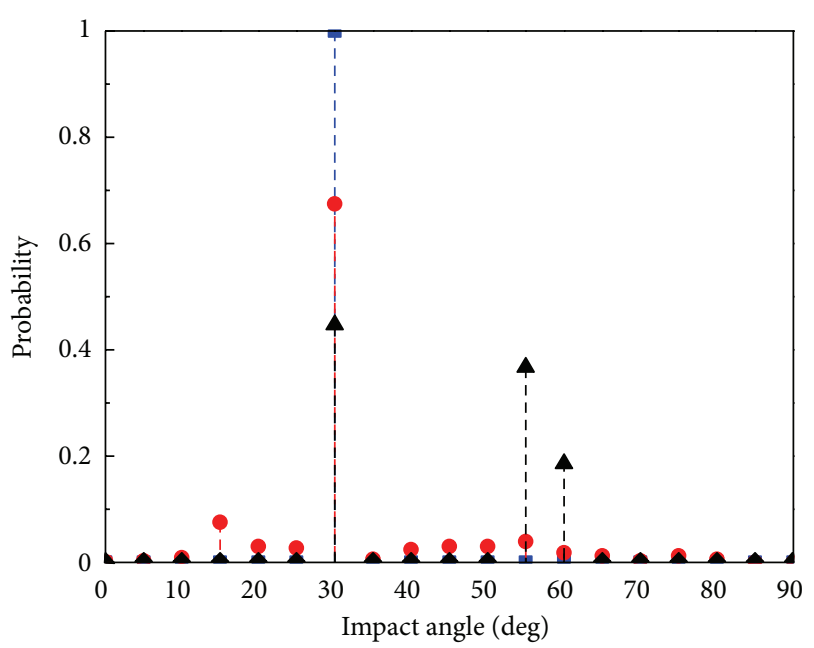

- Smooth blade

- Convex blade

$\Delta$ Groove blade

Figure 8: Particle-surface impact angle distribution.

surface, all the impact angles were concentrated in $30^{\circ}$. On the convex surface, the impact angles are distributed in the range of $0-90^{\circ}$, and more than $60 \%$ probability impact angles are in the range of $25-30^{\circ}$. On the other hand, for the groove surface, most of the impact angles fall in the range of 50-65. Therefore, compared with the smooth and convex surfaces, the impacts on the groove surface occurred at the high impact angles which lead to lower erosion. This is another reason why the groove surface exhibits more erosion resistance [16].

3.5. Analysis of Particle Trajectories and Collision Times. Figures 9, 10, and 11 are the trajectories of 10 particles from the incident source under given simulation conditions.

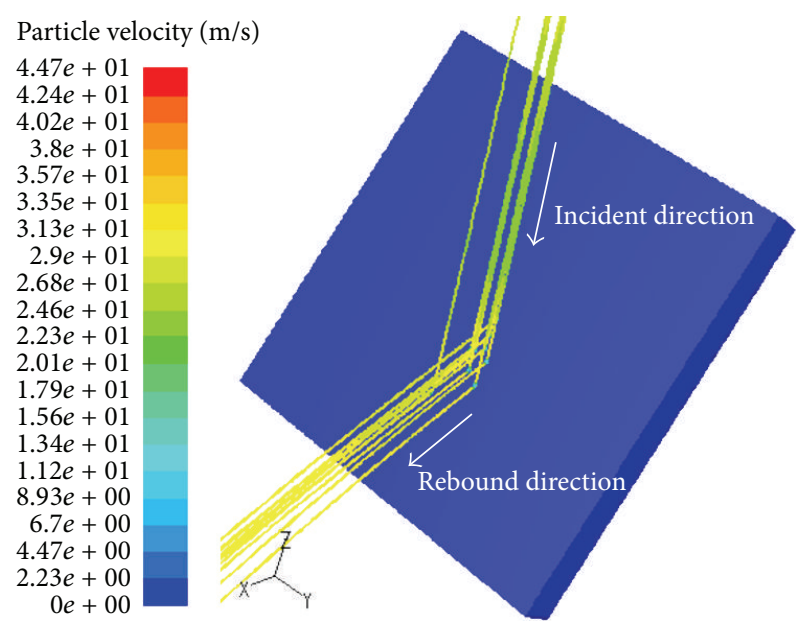

Figure 9: The trajectories of particle collision smooth surface.

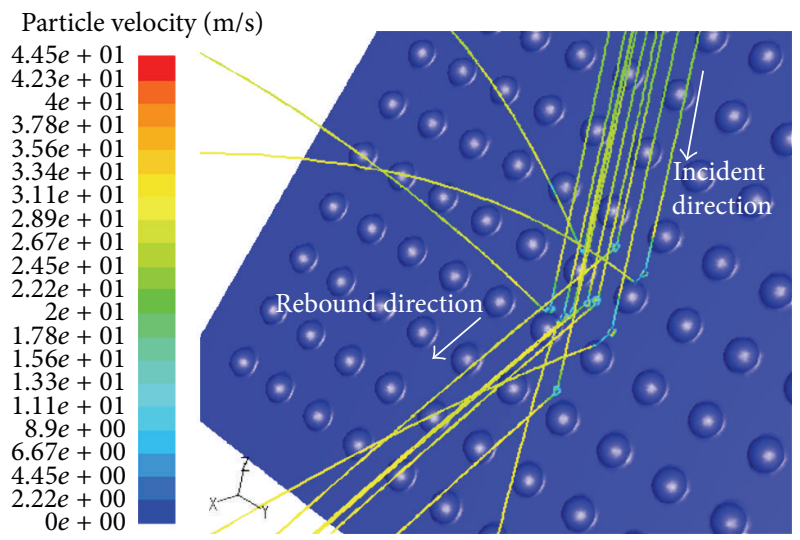

FIGURE 10: The trajectories of particle collision convex surface.

The sampling analysis and statistic shows that the collision number of 4240 particles from the incident source with smooth blade, convex blade, and groove blade, respectively, 291, 332, and 452. Obtained by calculating the numbers of collisions on the three-blade surfaces per unit area were 0.116 , 0.115 , and $0.113 / \mathrm{mm}^{2}$.

It can be seen that the impact times of groove and convex surfaces were lower than that of smooth surface, and the groove surface showed the lowest impact times. Hence, the reduction of impact on the groove surface can lead to the decrease of erosion wear to a certain degree.

\section{Conclusions}

In the present work continuous-discrete phase models are used to predict the erosion of the three kinds of blades. Conclusions are as follows.

The groove surface blades showed the best erosion resistance compared to other two kinds of blades. The flow velocities are higher around the surface of smooth surface than those of the convex surface and the groove surface from the velocity contours. The groove surface has a great influence 


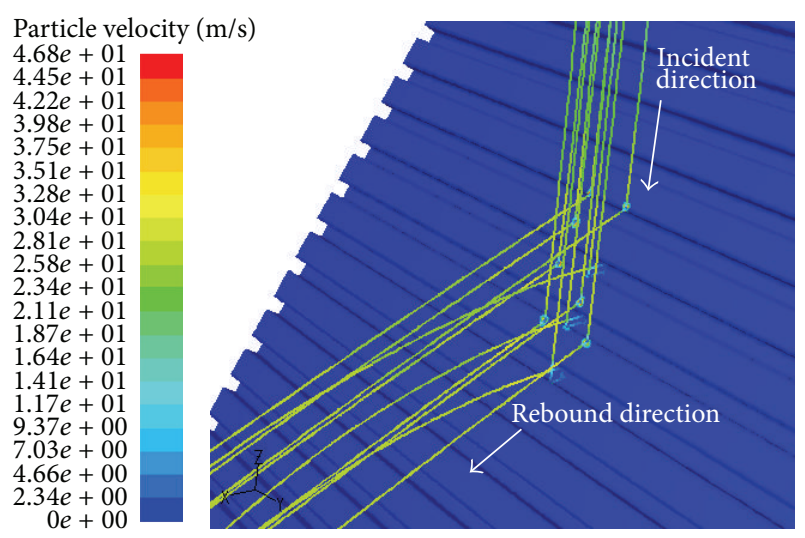

FIGURE 11: The trajectories of particle collision groove surface.

on the airflow. The particle impact velocity of biomimetic groove axial fan blade is less than smooth blade and the convex blade. The impacts on the biomimetic groove axial fan blade occurred at the high impact angles which are relatively less susceptible to impact damage compared with the smooth and convex surfaces, while the surface smooth and convex hull-shaped biomimetic form of axial fan blades collision occurred in apt erosion low-angle region. The impact times of groove and convex surfaces were lower than those of smooth surface, and the groove surface showed the lowest impact times.

\section{Conflict of Interests}

The authors declare that there is no conflict of interests regarding the publication of this paper.

\section{Acknowledgment}

This work was supported by the Natural Science Foundation of China (nos. 51175220 and 51205161), Specialized Research Fund for the Doctoral Program of Higher Education (nos. 20100061110023 and 20120061120051), China Postdoctoral Science Foundation on the 51th Grant Program (2012M511345), the Projects of Cooperation and Innovation to National Potential Oil and Gas for Production and Research (no. OSR-04-04), the Scientific and the Technological Development Project of Jilin Province (no. 20130522066), and Basic Scientific Research Expenses of Project of Jilin University (450060481176).

\section{References}

[1] S. Oomen-Hurst, M. D. Abad, M. Khanna, and S. C. Veldhuis, "Comparative wear behavior studies of coated inserts during milling of NiCrMoV steel," Tribology International, vol. 53, pp. 115-123, 2012.

[2] G. R. Desale, C. P. Paul, B. K. Gandhi, and S. C. Jain, "Erosion wear behavior of laser clad surfaces of low carbon austenitic steel," Wear, vol. 266, no. 9-10, pp. 975-987, 2009.

[3] A. Patnaik, A. Satapathy, N. Chand, N. M. Barkoula, and S. Biswas, "Solid particle erosion wear characteristics of fiber and particulate filled polymer composites: a review," Wear, vol. 268, no. 1-2, pp. 249-263, 2010.

[4] A. Klimpel, L. A. Dobrzański, A. Lisiecki, and D. Janicki, “The study of properties of $\mathrm{Ni}-\mathrm{W}_{2} \mathrm{C}$ and $\mathrm{Co}-\mathrm{W}_{2} \mathrm{C}$ powders thermal sprayed deposits," Journal of Materials Processing Technology, vol. 164, pp. 1068-1073, 2005.

[5] A. Krella, "An experimental parameter of cavitation erosion resistance for TiN coatings," Wear, vol. 270, no. 3-4, pp. 252257, 2011.

[6] P. Sampathkumaran, C. Ranganathaiah, S. Seetharamu, and K. Kishore, "Chromium-manganese iron alloy system design cast in metal and sand moulds for erosion resistance: a positron lifetime study," International Journal of Advanced Manufacturing Technology, vol. 51, no. 1-4, pp. 45-52, 2011.

[7] J. Du, P. Zhang, J. J. Zhao, and Z. H. Cai, "Erosion-resistant PVD ZrAlCuN coating for titanium alloy," Advances in Composites, vol. 150-151, pp. 51-55, 2011.

[8] Z. Shaofeng, W. Yucheng, and H. Xinmin, "Preparation and properties of electroless deposited $\mathrm{Ni}-\mathrm{Zn}-\mathrm{P}-\mathrm{TiO}_{2}$ nanocomposite coating," Asian Journal of Chemistry, vol. 23, no. 5, pp. 2299-2302, 2011.

[9] G. C. Saha, T. I. Khan, and G. A. Zhang, "Erosion-corrosion resistance of microcrystalline and near-nanocrystalline WC17 Co high velocity oxy-fuel thermal spray coatings," Corrosion Science, vol. 53, no. 6, pp. 2106-2114, 2011.

[10] M. Suzuki, K. Inaba, and M. Yamamoto, "Numerical simulation of sand erosion phenomena in square-section 90-degree bend," Journal of Fluid Science and Technology, vol. 3, no. 7, pp. 868$880,2008$.

[11] V. Bonache, M. D. Salvador, J. C. García, E. Sánchez, and E. Bannier, "Influence of plasma intensity on wear and erosion resistance of conventional and nanometric WC-Co coatings deposited by APS," Journal of Thermal Spray Technology, vol. 20, no. 3, pp. 549-559, 2011.

[12] B. S. Mann, V. Arya, and B. K. Pant, "Influence of laser power on the hardening of $\mathrm{Ti}_{6} \mathrm{Al}_{4} \mathrm{~V}$ low-pressure steam turbine blade material for enhancing water droplet erosion resistance," Journal of Materials Engineering and Performance, vol. 20, no. 2, pp. 213-218, 2011.

[13] K. Nehete, R. A. Sharma, and L. Chaudhari, "Study of erosion resistance and mechanical properties of unsaturated polyester based nano-composites," IEEE Transactions on Dielectrics and Electrical Insulation, vol. 19, no. 2, pp. 373-382, 2012.

[14] C. Sanchez, H. Arribart, and M. M. G. Guille, "Biomimetism and bioinspiration as tools for the design of innovative materials and systems," Nature Materials, vol. 4, no. 4, pp. 277-288, 2005.

[15] P. Fratzl and F. G. Barth, "Biomaterial systems for mechanosensing and actuation," Nature, vol. 462, no. 7272, pp. 442-448, 2009.

[16] H. Zhiwu, Z. Junqiu, G. Chao, W. Li, and L. Ren, "Erosion resistance of bionic functional surfaces inspired from desert scorpions," Langmuir, vol. 28, no. 5, pp. 2914-2921, 2012.

[17] Z. Han, J. Zhang, C. Ge et al., "Anti-erosion function in animals and its biomimetic application," Journal of Bionic Engineering, vol. 7, pp. S50-S58, 2010.

[18] A. Campos-Amezcua, A. Gallegos-Muñoz, C. A. Romero, Z. Mazur-Czerwiec, and R. Campos-Amezcua, "Numerical investigation of the solid particle erosion rate in a steam turbine nozzle," Applied Thermal Engineering, vol. 27, no. 14-15, pp. 2394-2403, 2007. 
[19] A. A. Hamed, W. Tabakoff, R. B. Rivir, K. Das, and P. Arora, "Turbine blade surface deterioration by erosion," Journal of Turbomachinery, vol. 127, no. 3, pp. 445-452, 2005.

[20] A. Hamed, W. Tabakoff, and R. Wenglarz, "Erosion and deposition in turbomachinery," Journal of Propulsion and Power, vol. 22, no. 2, pp. 350-360, 2006.

[21] J.-Q. Zhang, Z.-W. Han, W. Yin, H. Wang, C. Ge, and J. Jiang, "Numerical experiment of the solid particle erosion of bionic configuration blade of centrifugal fan," Acta Metallurgica Sinica, vol. 26, no. 1, pp. 16-24, 2013. 

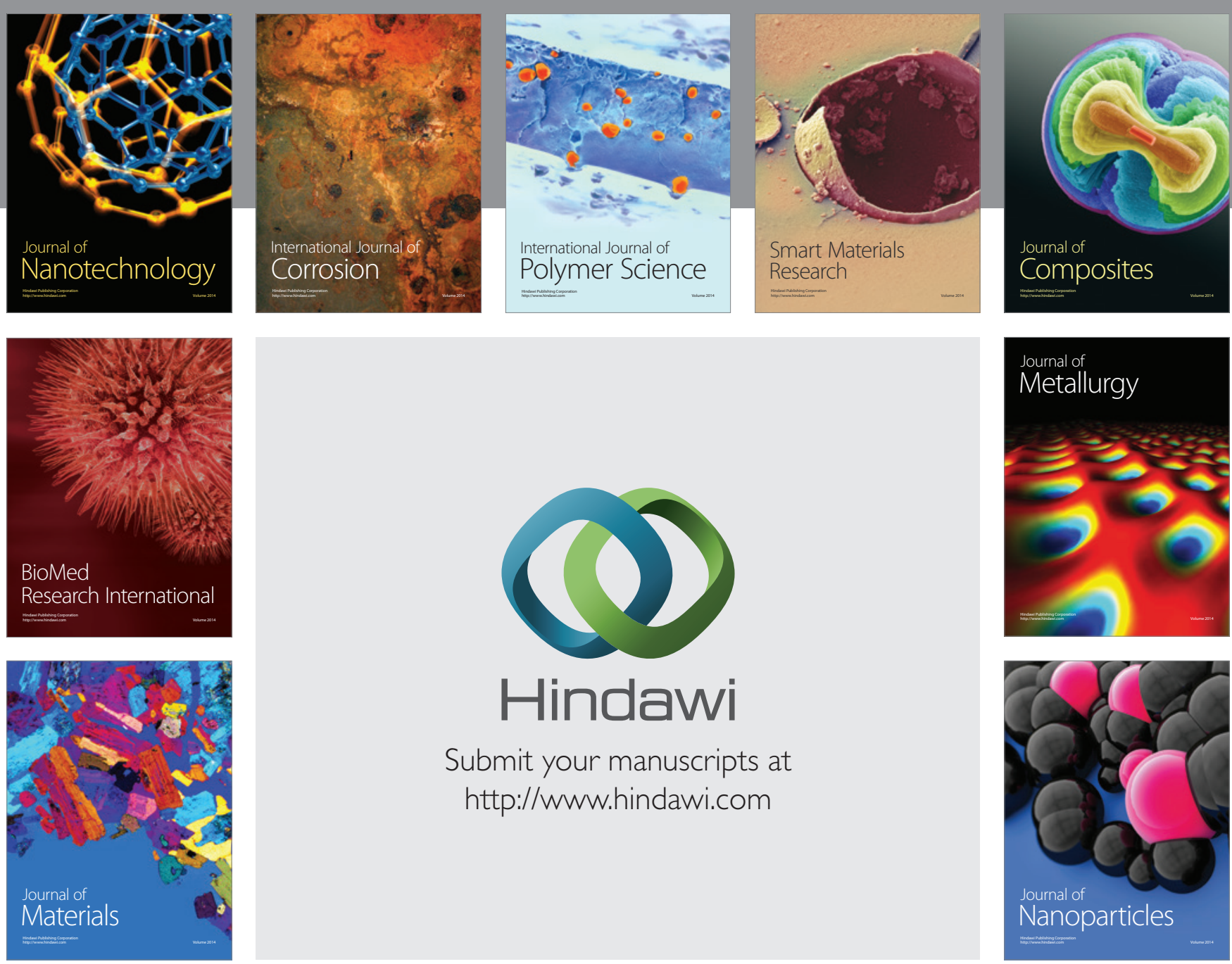

Submit your manuscripts at http://www.hindawi.com
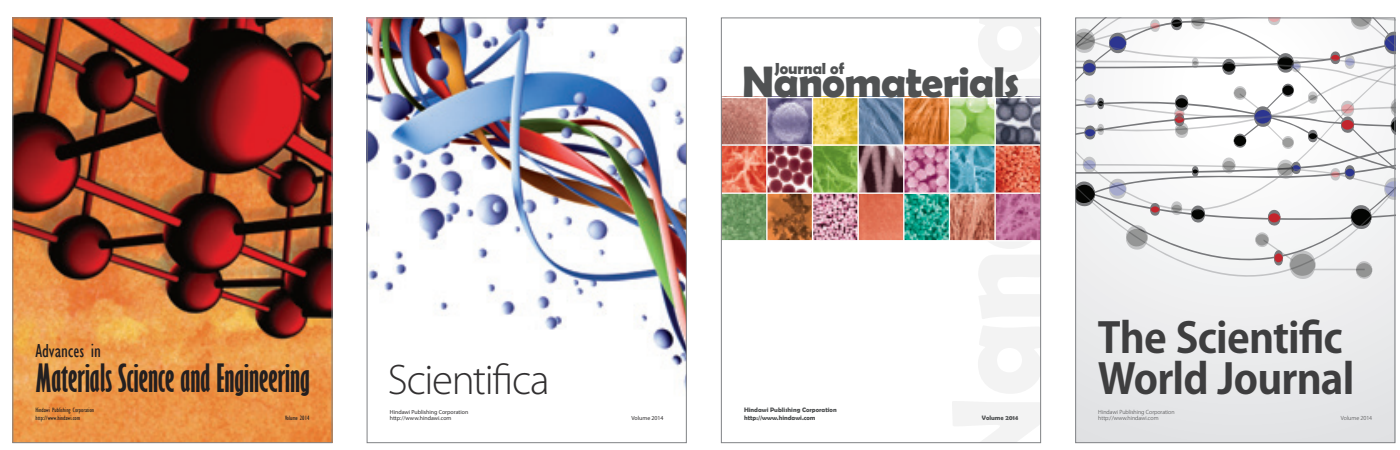

\section{The Scientific World Journal}
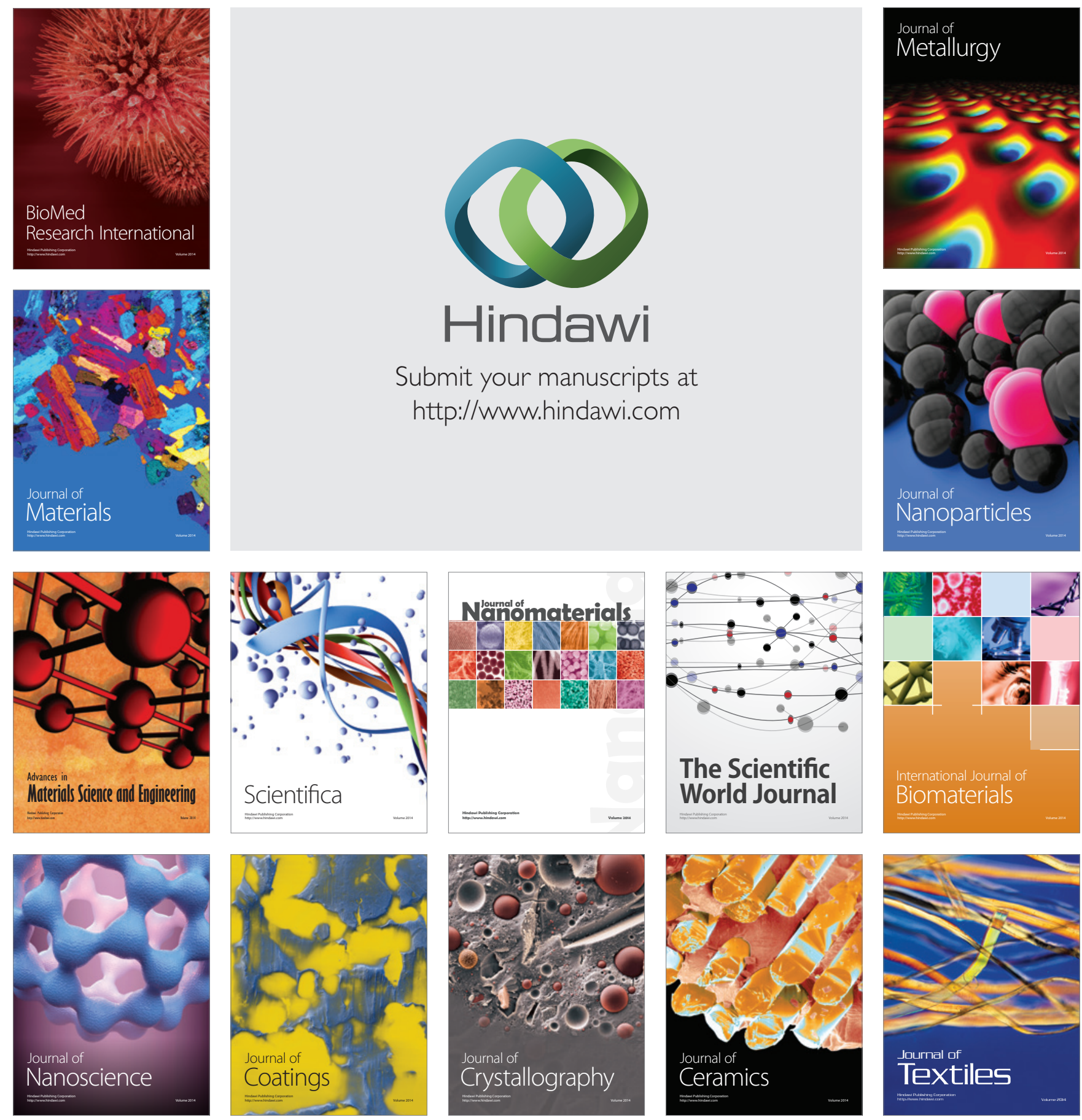